Gebhardt, Michael; Nestmann, Franz; Schweizerhof, Karl; Kemnitz, Bernhard Grundlagen für die hydraulische und statische Bemessung von wasser- und luftgefüllten Schlauchwehren

Verfügbar unter / Available at:

https://hdl.handle.net/20.500.11970/100640

Vorgeschlagene Zitierweise / Suggested citation:

Gebhardt, Michael; Nestmann, Franz; Schweizerhof, Karl; Kemnitz, Bernhard (2008): Grundlagen für die hydraulische und statische Bemessung von wasser- und luftgefüllten Schlauchwehren. In: Wasserwirtschaft J. 98 (3/2008). S. 27-32. 


\title{
Autorenfassung
}

Gebhardt, Nestmann, Schweizerhof, Kemnitz: Grundlagen für die hydraulische und statische Bemessung von wasser- und luftgefüllten Schlauchwehren, 2008

Erstveröffentlichung in WasserWirtschaft 3 (2008), S. 27-32.

Für eine korrekte Zitierbarkeit ist die Seitennummerierung

der Originalveröffentlichung für jede Seite kenntlich gemacht.

\section{Grundlagen für die hydraulische und statische Bemessung von wasser-und luftgefüllten Schlauchwehren}

\author{
Michael Gebhardt, Franz Nestmann, Karl Schweizerhof und Bernhard Kemnitz
}

Schlauchwehre besitzen bei Bau, Betrieb und Instandhaltung wirtschaftliche Vorteile gegenüber Stahlwasserbauverschlüssen, weshalb dieser Wehrtyp in den vergangenen Jahren an Bedeutung gewonnen hat. In diesem Beitrag werden die Grundlagen für die hydraulische Bemessung vorgestellt, welche das Ergebnis umfangreicher Modelluntersuchungen zur Überströmungscharakteristik und zur Schwingungsempfindlichkeit sind. Bei der statischen Bemessung geht es neben der Berechnung der Membrankräfte auch um die Eignung analytischer Verfahren zur Bestimmung der Schlauchgeometrie.

\section{Einleitung}

In der Wasser- und Schifffahrtsverwaltung des Bundes (WSV) werden 280 Wehranlagen betrieben, von denen die Hälfte älter als 50 Jahre ist. In den nächsten Jahren besteht folglich ein hoher Sanierungsbedarf bei immer knapper werdenden Haushaltsmitteln. Stahlwasserbauverschlüsse haben zwar eine hohe Lebensdauer von bis zu 70 Jahren, müssen aber im Schnitt alle 15 bis 20 Jahre einer umfassenden Instandsetzung unterzogen werden. An zwei Staustufen hat man sich in der WSV jetzt dafür entschieden, auf eine andere Technologie zu setzen: Bei den Ersatzneubauten Marklendorf an der Aller und Bahnitz an der Unteren-Havel-Wasserstraße werden erstmalig an Bundeswasserstraßen Schlauchwehre als Wehrverschlüsse eingesetzt. Hier wurde ein Kostenvorteil bei den Investitionen und Unterhaltungskosten von 20 bis $25 \%$ gegenüber konventionellen Klappenwehren ermittelt [8], [10]. Neben dem wirtschaftlichen Aspekt gibt es aber auch eine Reiheweiterer Vorteile, die Schlauchwehre gegenüber Stahlwasserbauverschlüssen besitzen:

- Die Konstruktion ist einfach und ohne mechanische Teile (Drehgelenke, Lager), ohne Korrosions- und Dichtungsprobleme und ohne Bedarf an umweltbelastenden Schmierstoffen. Schlauchwehre reagieren unempfindlich gegenüber Setzungen und Erdbeben.

- Die bei herkömmlichen Stahlwasserbauverschlüssen vorhandenen wartungsintensiven Antriebe, wie Hydraulikzylinder, Elektrostellantriebe oder Ketten, entfallen. Schlauchwehre werden allein durch das Ein- und Ausleiten von Luft oder Wasser in den Schlauchkörper gesteuert.

- Der Aufwand für Aussparungen sowie Bewehrungen ist gering und die Krafteinleitung in den Wehrkörper gleichmäßig verteilt. Der Bau wird dadurch wesentlich erleichtert, insbesondere dann, wenn die vorhandene Betonkonstruktion mit einbezogen werden muss. 


\section{Autorenfassung}

Gebhardt, Nestmann, Schweizerhof, Kemnitz: Grundlagen für die hydraulische und statische Bemessung von wasser- und luftgefüllten Schlauchwehren, 2008

- Der Betrieb im Hochwasserfall ist sicher, da ein Schlauch immer gelegt werden kann. An einigen Anlagen wurde daher von den Genehmigungsbehörden auf die Einhaltung der (n-1)-Bedingung u. a. nach DIN 19700 verzichtet.

- Die Montage oder der Austausch der Schlauchmembrane erfolgt innerhalb von wenigen Wochen, so dass sich die Bauzeiten sowie die Zeiträume für Revision und Instandsetzung erheblich verkürzen.

Die Verwendung von gewebeverstärktem Elastomer als Konstruktionsmaterial stößt in weiten Bereichen jedoch noch auf Skepsis. Hinweise und Empfehlungen zur Planung beschränkten sich bisher zumeist auf die Angaben der Hersteller. Durch die eingehende ingenieurwissenschaftliche Bearbeitung dieser Thematik sollen Impulse gegeben werden, um den flexiblen Kontrollbauwerken mehr Aufmerksamkeit zu schenken als bisher [4].

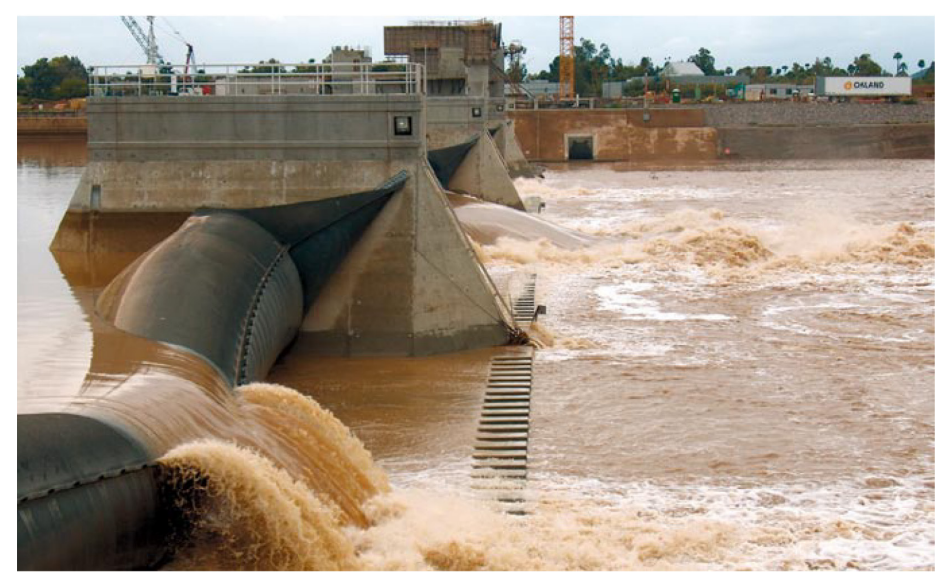

Bild 1: $\quad$ Luftgefülltes Schlauchwehr nach dem System Bridgestone am Tempe Town Lake, Arizona (4 Wehrfelder à 73,1 m Breite und 4,90 m Höhe) während eines Hochwassers im Februar 2005 [City of Tempe 2007]

Gebhardt, Nestmann, Schweizerhof, Kemnitz: Grundlagen für die hydraulische und statische Bemessung von wasser- und luftgefüllten Schlauchwehren. WasserWirtschaft 3 (2008), S. 27-32.

\section{Hydraulische Bemessung}

\section{1 Überströmungscharakteristik bei Luft- und Wasserfüllung}

Bei luftgefüllten Schlauchwehren tritt der Effekt auf, dass sie mit abnehmendem Innendruck nicht mehr gleichmäßig überströmtwerden, sondern an einer Stelle, zumeist im Bereich der Wehrwangen einknicken und eine lokale Abflusskonzentration auftritt (Bild 1). Die Ursache hierfür ist die Differenz zwischen Außen- und Innendruck aufder Oberwasserseite, die nicht, wie beim wasserge- 


\section{Autorenfassung}

Gebhardt, Nestmann, Schweizerhof, Kemnitz: Grundlagen für die hydraulische und statische Bemessung von wasser- und luftgefüllten Schlauchwehren, 2008

füllten Schlauch, konstant ist, sondern sich über die Wassertiefe ändert. Dadurch kann eine Instabilität des Systems auftreten, und die Membran wird gefaltet bzw. beult ein. Der Beginn des Einknickens liegt etwa bei $h_{\ddot{u}} / h_{o}=0,10$ bis 0,15 (Überfallhöhe $h_{\ddot{u}}$, Oberwassertiefe $h_{o}$ ). Durch die dabei auftretende V-förmige Eintiefung wird das Schlauchwehr einseitig beaufschlagt und die unterstromige Sohle lokal stärker beansprucht. Im Unterwasser können sich Walzensysteme ausbilden, die unter Umständen zu einer starken Beanspruchung der Uferböschungen führen. Nach praktischen Erfahrungen wird die Regelung des Oberwasserstands dadurch nicht beeinträchtigt [5].

In Bild 2 ist der Überfallbeiwert in Abhängigkeit von der dimensionslosen Überströmungshöhe $\mathrm{h}_{\ddot{u}} / \mathrm{h}_{\mathrm{o}}$ für ein wassergefülltes Schlauchwehr aufgetragen. Dabei ist zu erkennen, dass der Überfallbeiwert für kleine Überströmungshöhen am höchsten ist, da sich eine Unterdruckzone auf der Luftseite des Schlauchkörpers ausbildet und die Strömung dadurch zusätzlich beschleunigt wird. Der Überfallstrahllöst sich nicht ab, sondern „haftet“ quasi bis zum Fußpunkt des Schlauchkörpers an der Oberfläche. Bei Zylinderwehren kann sich wegen des Coanda-Effekts die Abflussleistung um 15 bis 20 \% erhöhen [3]. Mit steigender Überströmungshöhe löst sich die Strömung auf der Unterwasserseite ab, wobei der Ablösepunkt instabil ist und der Schlauchkörper durch die Druckschwankungen zu Schwingungen angeregt wird (s. Abschnitt 2.2).

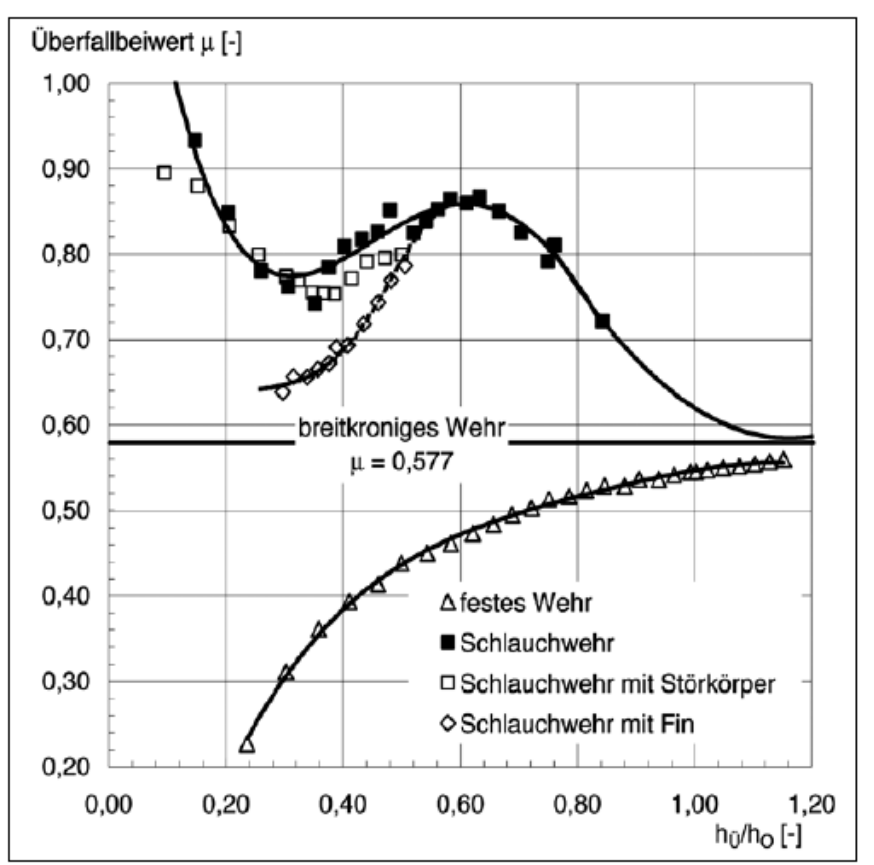

Bild 2: $\quad$ Überfallbeiwert $\mu$ für ein wassergefülltes Schlauchwehr ohne Abhilfemaßnahmen, mit Fin und mit Störkörperreihe bei vollkommenem Überfall $\left(\alpha_{0}=1,60 ;\left(l_{s}+l_{k}\right) / h_{o}=4,47\right.$, $\mathrm{h}_{\mathrm{o}}=$ const.) [4] 


\section{Autorenfassung}

Gebhardt, Nestmann, Schweizerhof, Kemnitz: Grundlagen für die hydraulische und statische Bemessung von wasser- und luftgefüllten Schlauchwehren, 2008

\subsection{Ursachen von Schwingungen}

Die Untersuchungen zum Schwingungsverhalten erfolgten an einem Vollmodell und an einem Ausschnittsmodell im Labor der Bundesanstalt für Wasserbau in Karlsruhe (Bild 3): Im Vollmodell wurde die Geometrie eines vollständigen Wehrfeldes mit einer Breite von etwa 1,70 m und einer Schlauchhöhe von 0,18 m abgebildet. Mit Hilfe eines Triangulationslasers wurde die Vertikalbewegung des Scheitelpunkts gemessen und anschließend mit einer Fast-Fourier-Transformation (FFT) ausgewertet. Die Auswahl der Modellmembranefür die beiden Modelle erfolgte nach dem CauchyKriterium, wobei der E-Modul in Zugversuchen an Probekörpern aus handelsüblichen Schlauchund Modellmembranen näherungsweise ermittelt wurde. Die Cauchy-Zahl berechnet sich dabei wie folgt:

$$
\mathrm{Ca}=\frac{\mathrm{v}^{2}}{\mathrm{E} \cdot \mathrm{I} /\left(\rho_{\mathrm{w}} \cdot \mathrm{h}_{\mathrm{s}}^{3}\right)}[-]
$$

$\mathrm{V} \quad$ Fließgeschwindigkeit $[\mathrm{m} / \mathrm{s}]$

E.I Biegesteifigkeit der Membran mit

$\mathrm{I}=1 / 12 \cdot \mathrm{ds}_{\mathrm{s}}^{3}\left[\mathrm{~N} \cdot \mathrm{m}^{2}\right]$

$\mathrm{h}_{\mathrm{s}} \quad$ Schlauchhöhe $[\mathrm{m}]$

$\mathrm{d}_{\mathrm{s}} \quad$ Dicke der Schlauchmembran [m]

$\rho_{\mathrm{w}} \quad$ Dichte Wasser $\left[\mathrm{kg} / \mathrm{m}^{3}\right]$

Die Modelluntersuchungen zeigen, dass Schlauchwehre aufgrund ihrer elastischen Struktur zu Schwingungen angeregt werden können, wie Schadensfälle am Sunbury-Damm [6], [7] oder am Mangla-Damm [2] zeigen. Problematisch sind dabei dauerhafte, also periodisch auftretende Schwingungen, bei denen sich der Schlauchkörper infolge der großen Verformungen an der Betonoberfläche aufscheuern kann oder bei denen die Zugfestigkeit der Membran, der Gewebeeinlagen oder der Befestigungskonstruktion durch die dynamische Belastung überschritten wird.

Die Grenze $h_{s} / h_{o}$, ab der ein Schlauchwehr in Resonanzschwingungen gerät, hängt maßgeblich von der Überströmungshöhe und vom Unterwasserstand ab. Grundsätzlich kann hier zwischen zwei selbsterregten Schwingungsarten unterschieden werden: Die erste Schwingungsart wird bei vergleichsweise niedrigen Unterwasserständen durch einen instabilen Ablösepunkt des Überfallstrahls und die dadurch resultierenden Druckschwankungen auf der Unterwasserseite des Schlauchkörpers induziert. Der Schlauchkörper verformt sich dabei zunächst in Fließrichtung, wobei dieser in vertikaler Richtung komprimiert und die Membranunterseite auf den Ablagetisch gedrückt werden. Bei der zweiten Schwingungsart wird der Fließquerschnitt bei der Überströmung des 


\section{Autorenfassung}

Gebhardt, Nestmann, Schweizerhof, Kemnitz: Grundlagen für die hydraulische und statische Bemessung von wasser- und luftgefüllten Schlauchwehren, 2008

Gebhardt, Nestmann, Schweizerhof, Kemnitz: Grundlagen für die hydraulische und statische Bemessung von wasser- und luftgefüllten Schlauchwehren. WasserWirtschaft 3 (2008), S. 27-32.
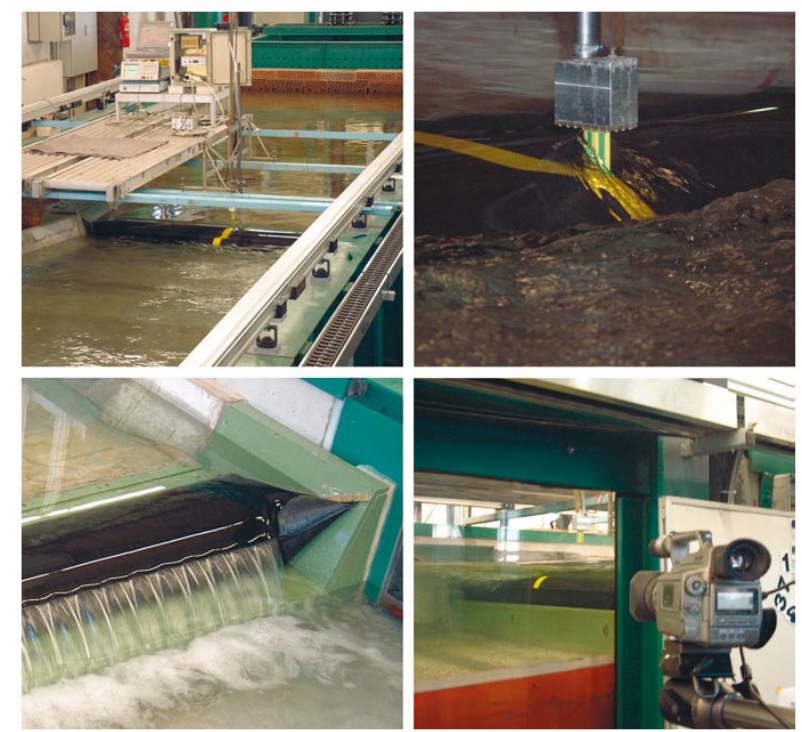

Bild 3: Vollmodell im Maßstab ca. 1:13: Blick vom Unterwasser (oben links), Triangulationslaser zur Messung der Vertikalamplituden (oben rechts), wassergefülltes Modell mit Fin (unten links) und Videodokumentation (unten rechts)

teilabgelegten Schlauchs eingeschnürt, und es entsteht ein Unterdruckbereich auf der Membranoberseite. Die resultierende Querkraft, die senkrecht zur Anströmrichtung wirkt, lenkt den Schlauchkörper nach oben aus und bewirkt eine Veränderung des Strömungsfelds über dem Schlauch (stationärer Quertrieb). Daraus ergibtsich eine Rückkopplung von der Strömung auf den Schlauchkörper: Der Formwiderstand nimmt zu, und der Schlauch wird wieder nach unten gedrückt. Die Auslenkung erfolgt dabei im Wesentlichen in vertikaler Richtung und mit niedrigerer Frequenz als bei der ersten Schwingungsart.

Mit Hilfe eines 3-D-Graphen (Bild 4) lässt sich aus den Messergebnissen für die zwei dimensionslosen Einflussgrößen Unterwasserstand $h_{u} / h_{o}$ und Schlauchhöhe $h_{s} / h_{o}$ der Bereich eingrenzen, in dem die maximalen Amplituden auftreten. Hier zeigt sich, dass Resonanzschwingungen nur in einem vergleichsweise kleinen Bereich möglicher Verschlusshöhen auftreten:

$$
0,60 \leq \frac{h_{s}}{h_{o}} \leq 0,70
$$




\section{Autorenfassung}

Gebhardt, Nestmann, Schweizerhof, Kemnitz: Grundlagen für die hydraulische und statische Bemessung von wasser- und luftgefüllten Schlauchwehren, 2008

Weiterhin ist festzustellen, dass der Schlauch bei einem höheren Unterwasserstand früher zu schwingen beginnt. Einen geringeren Einfluss haben der Innendruck, die Schlauchform und die Elastizität der Schlauchmembran.

\subsection{Abhilfemaßnahmen gegen Schwingungen}

Unter denselben hydraulischen Randbedingungen wurde die Wirksamkeit eines Deflektors am Beispiel der Fin nach dem System Bridgestone untersucht [4]. Die Lage der Fin in Umfangsrichtung ist durch die identischen Längen der oberen und unteren Membranteile vorgegeben. Die Länge der Fin $\mathrm{l}_{\mathrm{f}} \mathrm{im}$ aufgestellten Zustand beträgt etwa $\mathrm{l}_{\mathrm{f}} / \mathrm{h}_{\mathrm{o}}=0,04$. Im Vergleich zu dem Schlauchwehr ohne Abhilfemaßnahmen zeigt sich, dass die Fin eine stabile Ablösung des Überfallstrahls auf der Unterwasserseite bewirkt und dadurch die schwingungsinduzierenden Druckschwankungen verhindert. Gleichzeitig wird der Überfallstrahl bei kleinen Überströmungshöhen belüftet. Diese Ergebnisse entsprechen im Wesentlichen den Untersuchungsergebnissen von Ogihara und Muramatsu [6] am luftgefüllten Schlauch. Erst bei Unterwasserständen größer als $h_{u} / h_{o}>0,66$ sind periodische Schwingungen zu beob-

Gebhardt, Nestmann, Schweizerhof, Kemnitz: Grundlagen für die hydraulische und statische Bemessung von wasser- und luftgefüllten Schlauchwehren. WasserWirtschaft 3 (2008), S. 27-32.

achten (stationärer Quertrieb), allerdings mit kleinerer Amplitude als bei dem Modell ohne Fin (Bild 4 Mitte).

Als alternative Abhilfemaßnahme zur Vermeidung von Schwingungen wurde erstmalig im Modell eine Reihe von auf das Schlauchwehr aufgesetzten Störkörpern untersucht, deren Abstand und Form sich an den Strahlaufreißern für Klappenwehre orientierte. Dabei zeigte sich im Modell auf den ersten Blick ein etwas überraschendes Ergebnis: Die positive Wirkung der Störkörper beschränkte sich nicht nur auf kleine Überströmungshöhen und niedrige Unterwasserstände (also den Bereich, für den Strahlaufreißer bei Klappen eigentlich vorgesehen sind), sondern auch auf die Schwingungen infolge der Druckschwankungen auf der Unterwasserseite (Bild 4 rechts). Die Ursache hierfür ist, dass durch die Ablösungen an den Störkörpern Wirbel entstehen, die zu einer starken Erhöhung der Turbulenz in der Strömung führen. Damit werden die Druckschwankungen, die im Wesentlichen aus der radialen Umströmung resultieren, durchmischtund Resonanzschwingungen verhindert [4]. Für die weitere Optimierung der Störkörper wurden von der BAW und der Firma Floecksmühle Energietechnik GmbH im Jahr 2006 In-situ-Messungen in der Natur durchgeführt und die Wirkung einer modifizierten Form mit verschiedenen Abständen untersucht. Die Ergebnisse führten zu einer Anordnung der Störkörper, wie sie beim Ersatzneubau Bahnitz an der UnterenHavel-Wasserstraße realisiert wurde. 


\section{Autorenfassung}

Gebhardt, Nestmann, Schweizerhof, Kemnitz: Grundlagen für die hydraulische und statische Bemessung von wasser- und luftgefüllten Schlauchwehren, 2008

\section{Statische Bemessung}

\subsection{Eignung analytischer Berechnungsverfahren}

Bei konventionellen Stahlwasserbauverschlüssen kann der Abflussquerschnitt über geometrische Stellgrößen, wie z. B. Hubhöhe oder Lagewinkel, beschrieben werden. Bei Schlauchwehren hingegen ist eine Höhe nur schwer zu bestimmen, so dass hier der Innendruck als physikalische Stellgröße heranzuziehen ist. Verschiedene analytische Berechnungsansätze [1], [9], [12] wurden hierzu auf ihre Anwendbarkeit hin untersucht, die Algorithmen zur Berechnung mit der mathematischen Software MAPLE programmiertund die Geometrien für verschiedene Innendruckkoeffizienten berechnet.

\subsection{Bestimmung der Schlauchgeometrie}

Bei den drei verwendeten Verfahren nach der Membrantheorie wird das Eigengewicht

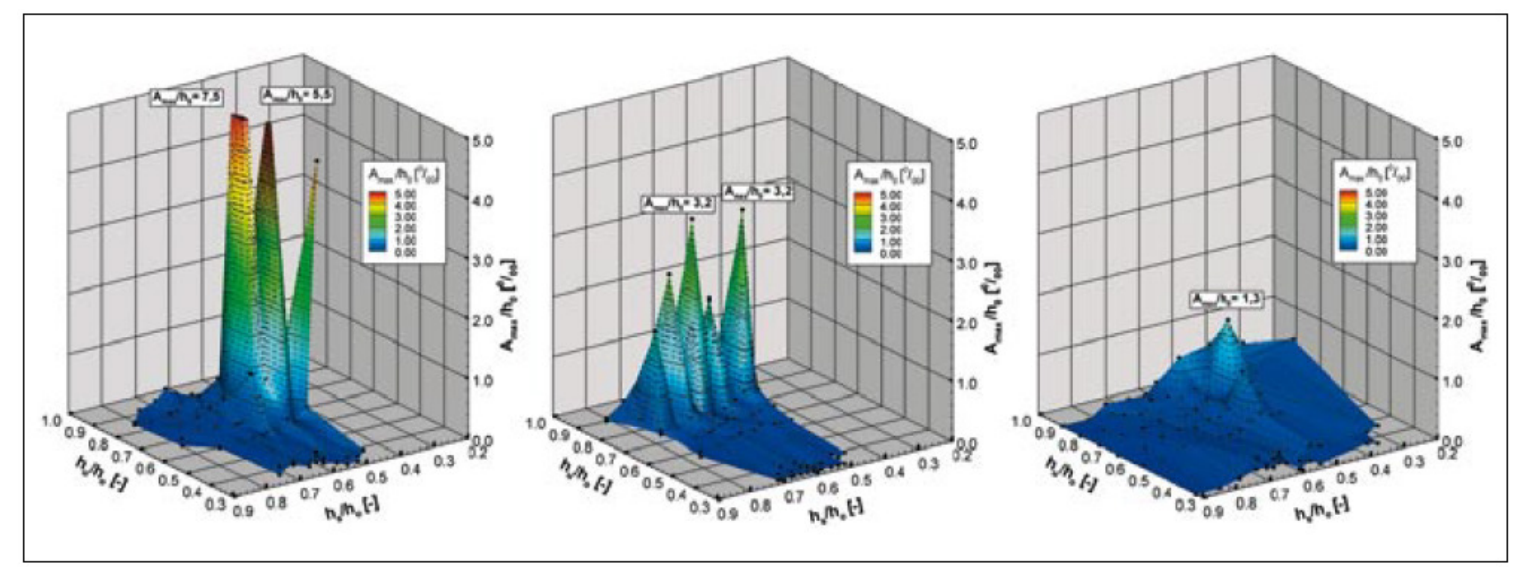

Bild 4: $\quad$ Maximale Amplituden $A_{\max } / h_{o}$ in Abhängigkeitvon Schlauchhöhe $\left(\mathrm{h}_{\mathrm{s}} / \mathrm{h}_{\mathrm{o}}\right)$ und Unterwasserstand $\left(\mathrm{h}_{\mathrm{u}} / \mathrm{h}_{\mathrm{o}}\right)$ - ohne Abhilfemaßnahmen (links), mit Deflektor (Mitte) und mit Störkörperreihe (rechts) [4] 


\section{Autorenfassung}

Gebhardt, Nestmann, Schweizerhof, Kemnitz: Grundlagen für die hydraulische und statische Bemessung von wasser- und luftgefüllten Schlauchwehren, 2008

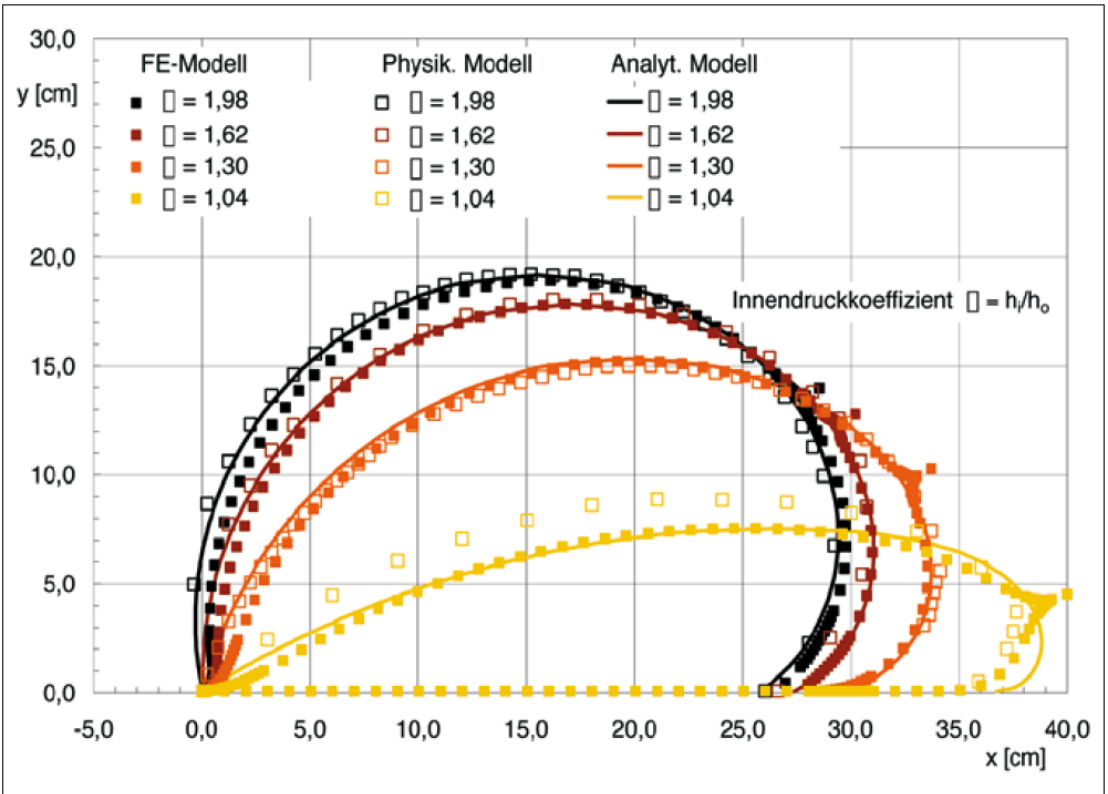

Bild 5: $\quad$ Schlauchform bei Wasserfüllung, nichtüberströmt - Vergleich von FE-Modell, physikalischem Modell und analytischer Berechnung [4]

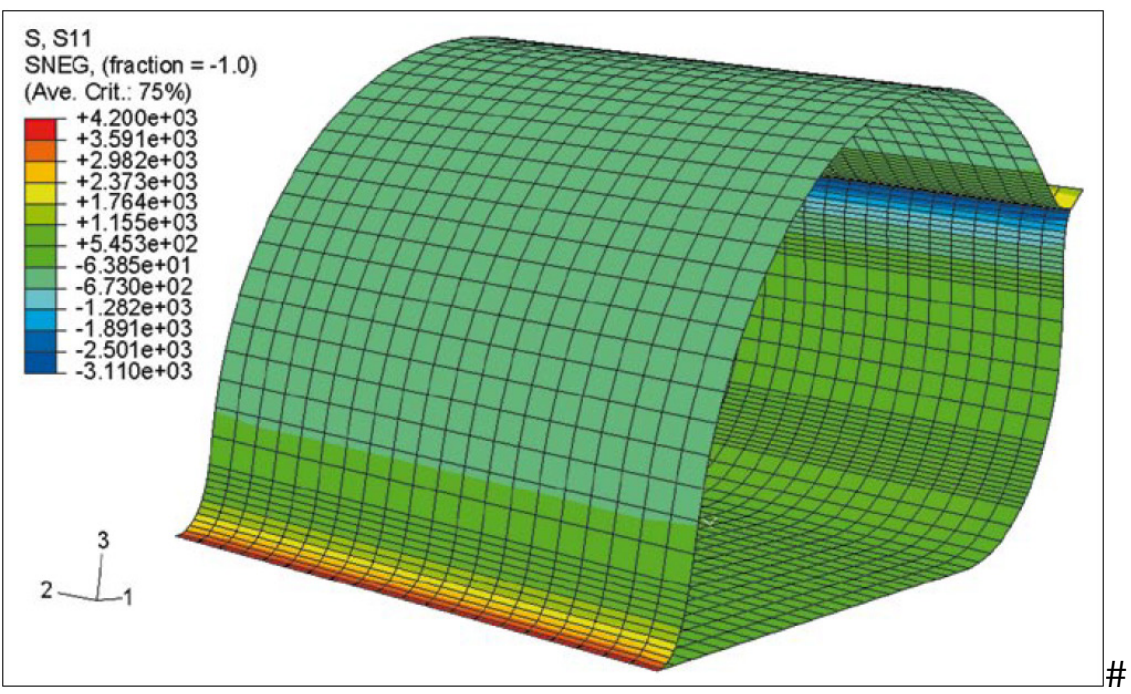

Bild 6: Spannungsverteilung in der Membran eines wassergefüllten Schlauchwehrs mit einer Befestigungsschiene $\left(\alpha_{0}=1,60 ; h_{s} / h_{0}=1,0\right)[4]$ 


\section{Autorenfassung}

Gebhardt, Nestmann, Schweizerhof, Kemnitz: Grundlagen für die hydraulische und statische Bemessung von wasser- und luftgefüllten Schlauchwehren, 2008

Gebhardt, Nestmann, Schweizerhof, Kemnitz: Grundlagen für die hydraulische und statische Bemessung von wasser- und luftgefüllten Schlauchwehren. WasserWirtschaft 3 (2008), S. 27-32.

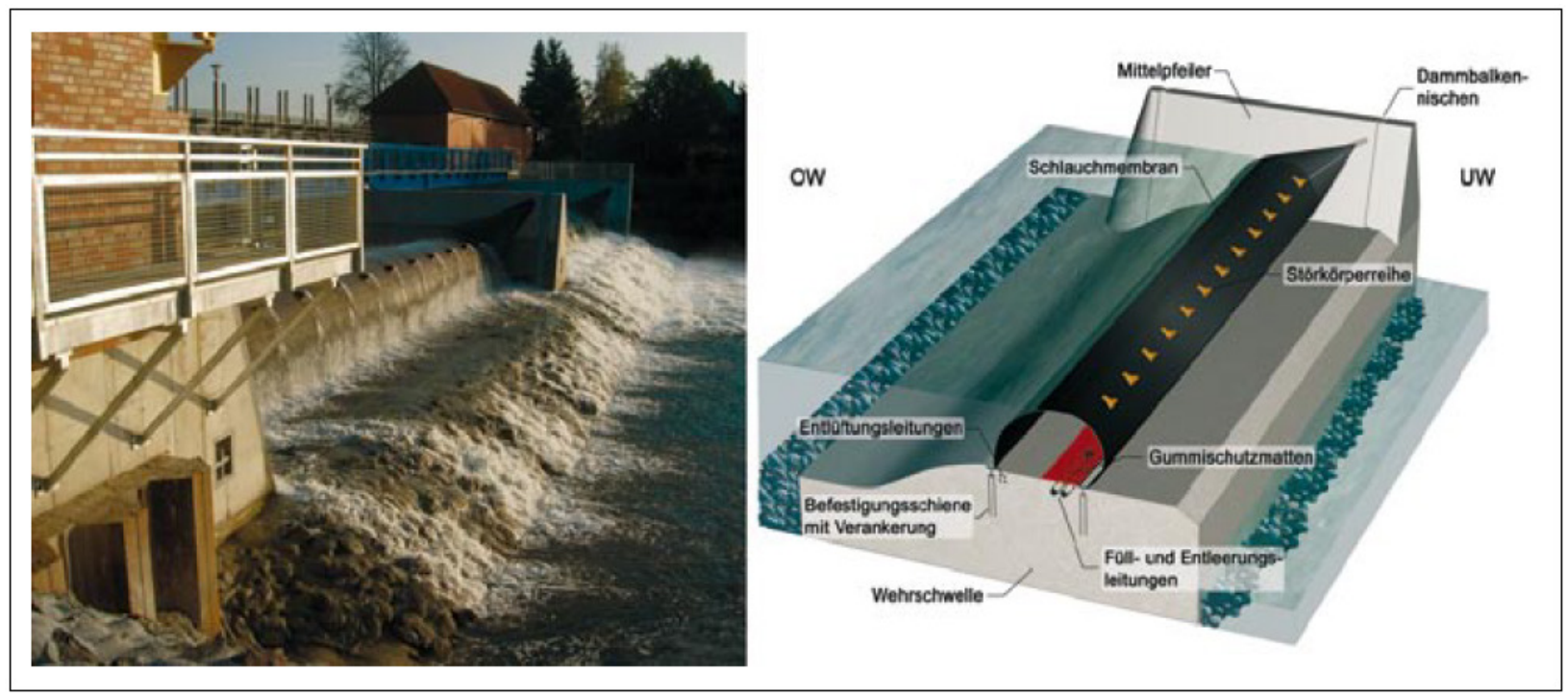

Bild 7: $\quad$ Allerwehr Marklendorf nach der Inbetriebnahme im Dezember 2006 [WSA Verden 2006] und isometrische Ansicht des rechten Wehrfeldes

und die Dehnung der Membran vernachlässigt sowie eine hydrostatische Druckverteilung entlang der Schlauchoberflächezugrundegelegt. Da es sich im Wesentlichen um ein ebenes Problem handelt, erfolgt die Berechnung nur im Querschnitt, was dem Spannungszustand an einem unendlich langen Rohr entspricht. Die so berechneten dimensionslosen Koordinaten werden nach Watson [12] auch als „complete dams“, also vollständige Schlauchformen, bezeichnet. Sie treffen auf Schlauchkörper mit einer Befestigungsschiene zu (Randbedingung im Sinne der Membrantheorie) und weisen aus Gleichgewichtsgründen auf der Luftseite eine horizontale Tangente auf.

Die berechneten Schlauchformen zeigen bei Wasserfüllung für den Fall ohne Überströmung und ohne Unterwassereinfluss eine sehr gute Übereinstimmung mit den Modellversuchen, wie dies aus Bild 5 ersichtlich ist. Lediglich für den Versuch mit einem Innendruckkoeffizienten von $\alpha=1,04$ ergeben sich Abweichungen von $15 \%$. Diese große Differenz ist darauf zurückzuführen, dass sich für $\alpha \rightarrow 1,00$ sowohl Modellversuch als auch Berechnungsverfahren in einem Grenzbereich befinden: Hier gibt es keine physikalische und keine numerische Lösung, da ein minimaler Überduck vorhanden sein muss, damit der Schlauchkörper nicht überströmt wird.

Das zum Vergleich herangezogene japanische Verfahren [9] berücksichtigt über einen vereinfachten hydrostatischen Ansatz zusätzlich die Überströmungund den Rückstau vom Unterwasser. Beim 


\section{Autorenfassung}

Gebhardt, Nestmann, Schweizerhof, Kemnitz: Grundlagen für die hydraulische und statische Bemessung von wasser- und luftgefüllten Schlauchwehren, 2008

Vergleich der berechneten und gemessenen Geometrien ergeben sich allerdings mit steigender Überströmungshöhe zunehmende Abweichungen. Hier zeigt sich, dass der dynamische Druckanteil bei der Berechnung nicht vernachlässigt werden kann und die Ermittlung von WasserstandAbfluss-Beziehungen im physikalischen Modellversuch erfolgen muss.

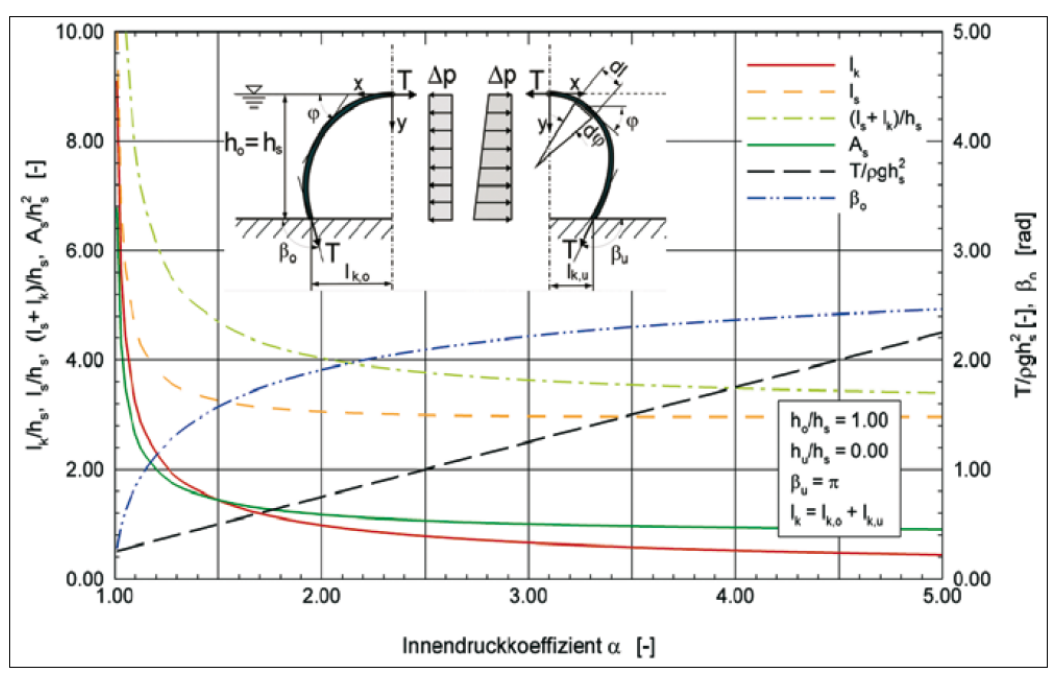

Bild 8: Membrankraft und geometrische Parameter für den wassergefüllten Schlauch, nicht überströmt und ohne Unterwassereinfluss [4]

\subsection{Dominierende Membrankraft für die Bemessung der Gewebeeinlagen}

Im Hinblick auf die in Abschnitt 3.2 beschriebenen Vereinfachungen zur Berechnung von Geometrie und Membrankraft nach der Membrantheorie kann bei Finite-Elemente-Verfahren (FE-Verfahren) die Steifigkeit der Schlauchmembran durch die Verwendung von Schalenelementen berücksichtigt werden. Hierzu wurden statische Analysen an verschiedenen FE-Modellen mit dem Programmpaket ABAQUS durchgeführt.

Das FE-Modell besteht aus zwei übereinander liegenden Membranen, die an der Unterwasserseite miteinander verbunden sind, und damit die Fin bilden. Das Konstruktionsprinzip hat für die FEBerechnung den Vorteil, dass im unbelasteten Zustand ein völlig spannungsfreier Zustand vorliegt. Jede Membran wird als dünne, isotrope, elastische Schale modelliert. Auf Basis der gemessenen Schlauchgeometrien im Modellversuch wurden das FE-Modell und insbesondere die dabei verwendete Diskretisierung, die Randbedingungen sowie das Werkstoffverhalten validiert.

In Bild 6 ist die Verteilung der Normalspannungen in Umfangsrichtung dargestellt. Während sich die Spannungsverteilung in Umfangsrichtung praktisch nicht ändert, können an den Befestigungsschienen und an der Fin die Spannungen in den äußersten Materialfasern die querschnittsgemittelte Spannung um das mehr als 10-fache übersteigen. Außerhalb der stark gekrümmten Bereiche kann hingegen über die Ermittlung der Primärschnittgrößen gezeigt werden, dass der Anteil der 


\section{Autorenfassung}

Gebhardt, Nestmann, Schweizerhof, Kemnitz: Grundlagen für die hydraulische und statische Bemessung von wasser- und luftgefüllten Schlauchwehren, 2008

Biegung gering ist und die Ermittlung der Membrankraft nach analytischen Verfahren ausreichend genau ist. Für die vollständigen Schlauchformen, in der Regel auch der Bemessungsfall, kann die Membrankraft T damit direkt berechnet werden:

- Wasserfüllung

$$
T=\frac{1}{4}(2 \alpha-1) \rho_{w} g h_{s}^{2} \text { mit } \alpha=\frac{h_{i}}{h_{o}}
$$

- Luftfüllung:

$$
T=\frac{1}{2} \alpha \rho_{w} g h_{s}^{2} \text { mit } \alpha=\frac{p_{i}}{\rho_{w} g h_{o}}
$$

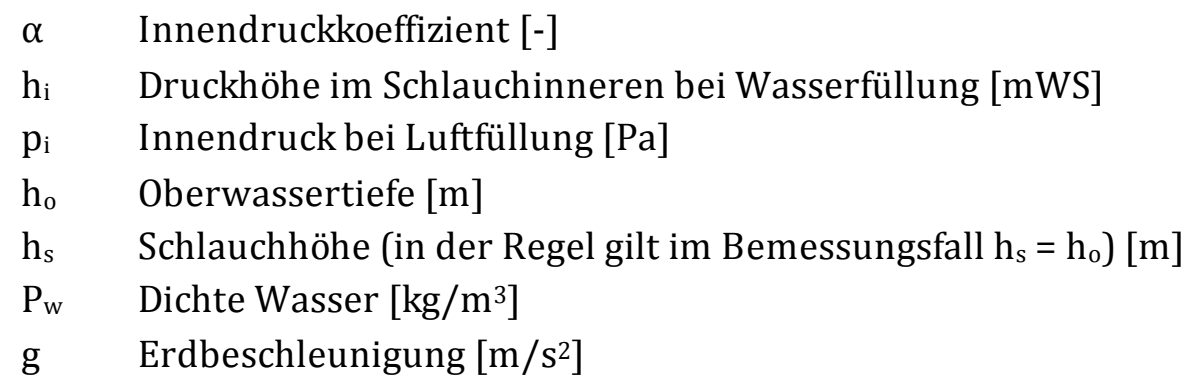

Gebhardt, Nestmann, Schweizerhof, Kemnitz: Grundlagen für die hydraulische und statische Bemessung von wasser- und luftgefüllten Schlauchwehren. WasserWirtschaft 3 (2008), S. 27-32.

\section{Anwendungsbeispiele}

Nach etwa zweieinhalbjähriger Bauzeit wurden die Schlauchverschlüsse der Wehranlage Marklendorf im Dezember 2006 durch das WSA Verden in Betrieb genommen (Bild 7). Die Fertigstellung des Ersatzneubaus für das Wehr Bahnitz an der Unteren Havel ist vom WNA Berlin für Sommer 2008 geplant. Die beiden Wehrebestehen aus je zwei Wehrfeldern von 23,60 m (Marklendorf) bzw. 36,50 m (Bahnitz) Breite und einer Höhe von ca. 2,20 m bzw. 2,40 m. Die Wehrfeldbreite sowie die Geometrie der Wehrschwelle und -pfeiler wurden von der BAW anhand von Modellversuchen optimiert. Aufgrund des besseren Regelungsverhaltens wurde für beide Schlauchwehre Wasser als Füllmedium empfohlen und als Alternative zur Fin eine Störkörperreihe vorgeschlagen, die bei etwa $90 \%$ der abgelegten Membran angeordnet ist. 


\section{Autorenfassung}

Gebhardt, Nestmann, Schweizerhof, Kemnitz: Grundlagen für die hydraulische und statische Bemessung von wasser- und luftgefüllten Schlauchwehren, 2008

\section{Zusammenfassung und Ausblick}

Mit Hilfe von systematischen Untersuchungen an physikalischen Modellen wurde gezeigt, dass Schlauchwehre ab einer bestimmten Überströmungshöhe zu Resonanzschwingungen angeregt werden können. Durch Abhilfemaßnahmen können diese vermieden oder erheblich reduziert werden. Eine Störkörperreihe erwies sich als wirkungsvolle Alternative zu einem Deflektor [4]. Störkörper wurden erstmalig an den beiden Anlagen in Marklendorf und Bahnitz realisiert. Aufgrund der geringeren Masse neigen luftgefüllte Schlauchwehre generell eher zu Schwingungen.

Der maßgebende Zustand für die statische Bemessung ist der Fall, dass der Schlauchkörper nicht überströmt wird: Die Differenz von Außen- und Innendruck entlang der Schlauchmembran ist dann am größten. Für viele Anwendungsfälle können damit Geometrie und Membrankraft in Abhängigkeit von Innendruck, Schlauchumfang, Ober- und Unterwasserstand sowie Befestigungsabstand berechnet und in Form von dimensionslosen Bemessungsdiagrammen dargestellt werden (Bild 8). Während die Lastabtragung bei Schlauchwehren in Feldmitteim Wesentlichen in Umfangsrichtung erfolgt, entstehen im Anschluss an die Wehrpfeiler bzw. -wangen Falten und dadurch zweiaxiale Spannungszustände. Für das Sturmflutwehr Ramspol in den Niederlanden wurde hier

Michael Gebhardt, Franz Nestmann, Karl Schweizerhof and Bernhard Kemnitz

Basic Principles for the Hydraulic and Structural Design of

Water- und Air-filled Inflatable Dams

Due to the simple design, operating and monetary advantages in comparison with steel gates, inflatable dams have become increasingly important in the past two decades. This paper deals with the basic principles for the hydraulic design. The results of extensive investigations with physical models serves as a foundation with the main focus of vibrations during operation. Vibrations can cause the rubber membrane to be abraded by the concrete surface, resulting in leaks, as cases of damage in the past have shown. The basic principles of the structural design cover not only the calculation of the dominating membrane force but also the applicability of analytical methods to calculate the dam shape.

ein Faktor von SCF = 4,2 (stress concentration factor) zur Berücksichtigung der Spannungsspitzen ermittelt [11]. Neben der Ermittlung statischer Spannungsspitzen ist unklar, wie hoch der dynamische Anteil, beispielsweise durch Turbulenzen in der Überströmung, durch Wellenbildung oder durch Querschwingungen sein kann. Fluid-Struktur-Modelle könnten hier in Zukunft Aufschluss über die Rückkopplung der Strömung auf das Schlauchwehr geben. 


\section{Autorenfassung}

Gebhardt, Nestmann, Schweizerhof, Kemnitz: Grundlagen für die hydraulische und statische Bemessung von wasser- und luftgefüllten Schlauchwehren, 2008

Zahlreiche Ausführungsbeispiele in der Welt zeigen, dass Schlauchwehre auch in großen Dimensionen möglich sind und eine kostengünstige Alternative zu konventionellen Stahlwasserbauverschlüssen darstellen. Daher gilt es, aus den Betriebserfahrungen zu lernen und die technischen Neuerungen in der Schlauchwehrtechnik zu nutzen und fortzuentwickeln.

\section{Autoren}

\section{Dr.-Ing. Michael Gebhardt BDir Bernhard Kemnitz}

Bundesanstalt für Wasserbau

Kussmaulstr. 17

76187 Karlsruhe

michael.gebhardt@baw.de

bernhard.kemnitz@baw.de

Univ.-Prof. Dr.-Ing. Dr. h. c. mult. Franz Nestmann

Bereich Wasserwirtschaft und Kulturtechnik

Institut für Wasser und Gewässerentwicklung

Universität Karlsruhe (TH)

Kaiserstraße 12

76131 Karlsruhe

Nestmann@iwg.uka.de

Prof. Dr.-Ing. Karl Schweizerhof

Institut für Mechanik

Universität Karlsruhe (TH)

Englerstraße 2

76131 Karlsruhe

Karl.Schweizerhof@ifm.uni-karlsruhe.de

\section{Literatur}

[1] Anwar, H. O.: Inflatable dams. In: Journal of the Hydraulic Division, ASCE 93 (1967), Mai, S. 99 119.

[2] Binnie, G. M. et al.: Inflatable weir used during construction of Mangla Dam. In: Proc. Instn. Civ. Eng. 54 (1974), Heft Nr. 7655, S.,625-639.

[3] Chanson, H.: Hydraulics of rubber dam overflow: A simple design approach. In: 13th, Australasian Fluid Mechanics Conference, Dezember 1998, S. 255-258. 


\section{Autorenfassung}

Gebhardt, Nestmann, Schweizerhof, Kemnitz: Grundlagen für die hydraulische und statische Bemessung von wasser- und luftgefüllten Schlauchwehren, 2008

[4] Gebhardt, M.: Hydraulische und Statische Bemessung von Schlauchwehren. In: Mitteilungen des Instituts für Wasser und Gewässerentwicklung - Bereich Wasserwirtschaft und Kulturtechnik - der Universität Karlsruhe (TH), Heft 235, 2006.

[5] Ittel, G.; Heimerl, S.: Innovative Sanierung der Wasserkraftanlage Kiebingen am Neckar. In: Wasserwirtschaft 91 (2001), Heft 9, S. 434-442.

[6] Ogihara, K.; Muramatsu, T.: Rubber dam, causes of oscillations of rubber dams and countermeasures. In: 21st IAHR Congress, Melbourne, Australia, Aug. 1985, S. 601-604.

[7] U. S. Army Corps of Engineers: Ice Engineering, Performance survey of inflatable dams in iceaffected waters. In: U. S. Army Research and Engineering Laboratory, Ice Engineering Information Exchange Bulletins, Hanover, New Hampshire, Oktober 2001, S. 1-5.

[8] Meine, G.: Allerwehr Marklendorf - Ein Gummischlauch als Stauverschluss. In: Der Ingenieur der Wasser- und Schifffahrtsverwaltung (2007), Heft 1.

[9] Ministry of Land, Infrastructure and Transport, River Bureau, Japan (Hrsg.): Technische Richtlinie für Schlauchwehre, 2000. Übersetzung aus dem Japanischen im Auftrag der Bundesanstalt für Wasserbau, unveröffentlicht.

[10] Reuter, R.: Ersatzneubau Wehr Bahnitz - Varianten, Planung und Bau eines Schlauchwehres. In: Mitteilungsblatt der BAW (2007), Heft 91.

[11] Van der Horst, A. Q. C; Rövecamp, N. H.: Design of the storm surge barrier Ramspol, The Netherlands. In: HTC Congress (2001).

[12] Watson, R.: A note on the shapes of flexible dams. In: Journal of Hydraulic Research, 23 (1985), Nr. 2, S. 179-194. 